\title{
Steering Wire Device
}

National Cancer Institute

\section{Source}

National Cancer Institute. Steering Wire Device. NCI Thesaurus. Code C50337.

A wire designed to connect to a steering device. 